\title{
Arterial Access Closure
}

National Cancer Institute

\section{Source}

National Cancer Institute. Arterial Access Closure. NCI Thesaurus. Code C100057.

A procedure to stop bleeding and close the location of arterial entry following a

diagnostic or therapeutic procedure. 